\title{
A multilevel logistic hidden Markov model for learning under cognitive diagnosis
}

\author{
Susu Zhang ${ }^{1}$ (D) Hua-Hua Chang ${ }^{2}$ \\ Published online: 7 May 2019 \\ (C) The Psychonomic Society, Inc. 2019
}

\begin{abstract}
Students who wish to learn a specific skill have increasing access to a growing number of online courses and opensource educational repositories of instructional tools, including videos, slides, and exercises. Navigating these tools is time-consuming and the search itself can hinder the learning of the skill. Educators are hence interested in aiding students by selecting the optimal content sequence for individual learners, specifically which skill one should learn next and which material one should use to study. Such adaptive selection would rely on pre-knowledge of how the learners' and the instructional tools' characteristics jointly affect the probability of acquiring a certain skill. Building upon previous research on Latent Transition Analysis and Learning Trajectories, we propose a multilevel logistic hidden Markov model for learning based on cognitive diagnosis models, where the probability that a learner acquires the target skill depends not only on the general difficulty of the skill and the learner's mastery of other skills in the curriculum but also on the effectiveness of the particular learning tool and its interaction with mastery of other skills, captured by random slopes and intercepts for each learning tool. A Bayesian modeling framework and an MCMC algorithm for parameter estimation are proposed and evaluated using a simulation study.
\end{abstract}

Keywords Learning $\cdot$ Diagnostic classification model $\cdot$ Hidden Markov model $\cdot$ Multilevel model

\section{Introduction}

With the increasing popularity of online and open-source education, such as massive online open courses (MOOCs), we start to see a plethora of online instructional tools targeting the learning of the same skill. Platforms such as OpenEd align instructional resources such as videos, games, and assessments to fine-grained learning objectives, such as the Common Core State Standards. Intelligent tutoring systems (Vanlehn, 2006) usually provide learners with a sequence

Electronic supplementary material The online version of this article (https://doi.org/10.3758/s13428-019-01238-w) contains supplementary material, which is available to authorized users.

Susu Zhang

sz2821@columbia.edu

Hua-Hua Chang

chang606@purdue.edu

1 Department of Statistics, Columbia University, 1255 Amsterdam Ave., New York, NY 10027, USA

2 Department of Educational Studies, Purdue University, 610 Purdue Mall, West Lafayette, IN 47906, USA of tasks for learning a target skill, with interactive feedback to aid problem solving and concept acquisition. A search on OpenEd for instructional tools targeting the learning of "Grade 7 Mathematics: Expressions and Equations" returned over 50 available videos, games, assessments, and so on.

With a massive amount of educational resources available online to the broad audience, people from different regions and backgrounds all receive the opportunity to take the same courses. However, due to the sheer volume of available materials, it is practically unfeasible for learners to navigate through all available materials for a course. A lot of MOOCs have reported having high attrition rate of registered students, usually with less than $10 \%$ of students who registered and completed the course. Using a survey for students who dropped out of an online course, Gütl et al. (2014) analyzed the potential reasons for the low retention rate of MOOCs. A majority of students suggested that insufficient time, unchallenging tasks, or overly difficult contents were part of the reasons for their drop-out. To maximize the students' interest and the learning efficiency with a limited amount of time, educators are interested in evaluating the effectiveness of different materials for a student and tailoring the course content to the student's level based on his or her needs. Such individualized learning is 
often achieved through an adaptive recommendation strategy (Chen, Li, Liu, \& Ying, 2017), where materials are sequentially selected and recommended to the student based on currently available information. Chen et al. (2017) suggested that a good recommendation strategy should utilize the information about both the learner and the instructional tool to maximize the gain (e.g., learning efficiency), thus in the present paper, we aim at developing a learning model that simultaneously estimates the student's progress over time and evaluates the efficiency of different learning materials.

At each stage of the learning process, a student's ability at that time point cannot be directly observed. Instead, it is often measured by test items following some kind of item response model, enabling us to measure the student's latent ability. Compared to item response theory models assuming one or a few continuous latent traits, cognitive diagnosis models assume students' mastery of different skills is discrete (e.g., dichotomous), hence allowing the computationally efficient simultaneous measurement of more finegrained skills in the curriculum. Therefore, in the current paper, we consider assessing the students' learning progress under the cognitive diagnostic modeling framework.

In the next sections of the paper, we will briefly introduce cognitive diagnosis models and hidden Markov models based on cognitive diagnosis models, provide an introduction of our current model, present a Bayesian estimation framework as well as the estimation algorithms, show the results from a simulation study on the proposed model, and, lastly, discuss the potential implications of the current model and some future directions.

\section{Learning models based on cognitive diagnosis}

Cognitive diagnosis models (CDMs), or diagnostic classification models (DCMs; Rupp, Templin, \& Henson, 2010), are restrictive latent class models measuring test-takers' mastery of various skills based on their responses to test questions/items. These models are designed to identify students' strengths and weaknesses in learning, providing guidance for personalized, targeted remedy and support. Under a CDM, a test-takers' underlying mastery status on a set of skills is usually denoted by a dichotomous vector $\boldsymbol{\alpha}=\left[\alpha_{1}, \ldots, \alpha_{K}\right]^{\prime}$, with $\alpha_{k}=1$ indicating mastery on skill $k$ and $\alpha_{k}=0$ indicating non-mastery. The probability of correct response on an item $j$ depends on the student's mastery status on attributes required by the item, captured by a $J \times K$ Q-matrix, with $q_{j k}=1$ if successful answering of item $j$ depends on the student's mastery of skill $k$, and 0 otherwise. How the probability of a correct response is influenced by the mastery of required attributes depends on the specific CDM. Many CDMs have been previously proposed (e.g., Junker \& Sijtsma, 2001; dela Torre, 2011; Henson, Templin, \& Willse, 2009; von Davier, 2008). One of the simplest and most commonly used CDM is the deterministic input, noisy-“and"-gate (DINA; e.g., Macready \& Dayton, 1977; Junker \& Sijtsma, 2001) model, under which, the probability of a correct response is given by

$$
P\left(X_{i j}=1 \mid \boldsymbol{\alpha}_{i}, s_{j}, g_{j}, \boldsymbol{q}_{j}\right)=\left(1-s_{j}\right)^{\eta_{i j}} g_{j}^{1-\eta_{i j}},
$$

where $\eta_{i j}=\prod_{k=1}^{K} \alpha_{i k}^{q_{j k}}$ is the ideal response, indicating whether subject $i$ possesses all required skills to answer item $j$ correctly, and $s_{j}$ and $g_{j}$ are the slipping and guessing parameters. Intuitively, the DINA model describes the case where a subject needs to have mastered all requisite skills of an item to be able to answer the item correctly with high probability $\left(1-s_{j}\right)$. Missing any of the item's requisite skills would result in a probability of a correct response of $g_{j}$ instead.

Whereas traditional research on CDMs focused on the assessment of the students' mastery at a single time point, there is increasing interest in assessing the students' progression of attribute mastery over time. Li, Cohen, Bottge, and Templin (2015) combined latent transition analysis (LTA; Langeheine, 1988; Collins \& Wugalter, 1992) with the DINA model to estimate the students' mastery change in a longitudinal setting. At each wave, a student has a probability of transitioning from non-mastery to mastery, or from mastery to non-mastery, on each skill. The transitions on different skills are assumed to be independent. Using the estimated transition probabilities between mastery and nonmastery on each skill between waves, they compared the effectiveness of two different learning interventions.

Chen et al. (2017) generalized Li et al. (2015) model to the case where attribute transitions are not necessarily independent - that is, instead of modeling the attribute-wise transitions and multiplying them to get the pattern-wise transition probabilities, they directly modeled the transition probabilities between different skill patterns. Different models for learning, including the most general unrestricted model and the monotonic model (i.e., where the probability of transitioning from mastery to non-mastery is 0 ), were introduced, and the cardinalities of the sets of all possible trajectories were derived.

Wang, Yang, Culpepper, and Douglas (2016) proposed the hidden Markov diagnostic classification model (HMDCM) with higher-order covariates affecting the learning outcome. Given the attribute pattern of subject $i$ at time $t$, $\boldsymbol{\alpha}_{i, t}=\left[\alpha_{i, 1, t}, \ldots, \alpha_{i, K, t}\right]^{\prime}$, the logit of the probability of transitioning from non-master to master on attribute $k$ is

$$
\begin{aligned}
\operatorname{logit}\left[P \left(\alpha_{i, k, t+1}=\right.\right. & \left.\left.1 \mid \alpha_{i, k, t}=0, \boldsymbol{\alpha}_{i, t}\right)\right] \\
= & \lambda_{0}+\lambda_{1} \theta_{i}+\lambda_{2} \sum_{\forall k^{\prime} \neq k} \alpha_{i, k^{\prime}, t} \\
& +\lambda_{3} \sum_{m=1}^{t} \sum_{j=1}^{J_{t}} q_{j, m, k} .
\end{aligned}
$$


In this model, $\theta_{i}$ was used to denote the overall, timeinvariant learning ability of subject $i$. The term $\sum_{\forall k^{\prime} \neq k} a_{i, k^{\prime}, t}$ represents how many attributes subject $i$ has already acquired other than attribute $k$, and $\sum_{m=1}^{t} \sum_{j=1}^{J_{t}} q_{j, m, k}$ denotes the number of items involving skill $k$ that the student has completed at previous time points, in other words, the amount of practice so far on attribute $k$. By using a higher-order logistic model for the transition probabilities in the hidden Markov model, the effect of different factors on the probability of learning a skill can hence be examined.

\section{Current model}

The higher-order hidden Markov modeling framework developed by Wang et al. (2016) provides a flexible tool for modeling the effect of different covariates, both of the learners and of the instructions, on the outcome of learning. We hence adopt this framework and formulate our current model as follows.

Similar to Wang et al. (2016), we assume that the learning process is monotonic, with the probability of transitioning from mastery to nonmastery equal to 0 for all time points and all skills. At a certain time point, $t \in\{0, \ldots, T\}$, a student $i \in\{1, \ldots, N\}$ is assigned to learn a skill $k \in\{1, \ldots, K\}$, by receiving an instructional tool $l \in$ $\left\{1, \ldots, L_{k}\right\}$ targeting the learning of skill $k$. Here, the set $\left\{1, \ldots, L_{k}\right\}$ is used to denote the collection of all instructional tools, including exercises, slides, videos, or games, that can have an effect on the learning of skill $k$. We let the attribute pattern of subject $i$ at time $t$ be $\boldsymbol{\alpha}_{i, t}$, then the probability that the subject masters skill $k$ at time $t+1$ is

$P\left(\alpha_{i, k, t+1}=1 \mid \boldsymbol{\alpha}_{i, t}, \boldsymbol{\gamma}_{k}, \mathbf{U}_{k, l}\right)= \begin{cases}1 & \alpha_{i, k, t}=1, \\ \frac{\exp \left(\lambda_{0, k, l}+\sum_{k^{\prime} \neq k} \lambda_{k^{\prime}, k, l} \cdot \alpha_{i, k^{\prime}, t}\right)}{1+\exp \left(\lambda_{0, k, l}+\sum_{k^{\prime} \neq k} \lambda_{k^{\prime}, k, l} \cdot \alpha_{i, k^{\prime}, t}\right)} & \alpha_{i, k, t}=0 .\end{cases}$

where $\lambda_{0, k, l}=\gamma_{0, k, 0}+U_{0, k, l}, \quad \lambda_{k^{\prime}, k, l}=$
$\gamma_{k^{\prime}, k, 0}+U_{k^{\prime}, k, l}, \quad$ with $\mathbf{U}_{k, l}=\left[\begin{array}{c}U_{0, k, l} \\ U_{1, k, l} \\ \vdots \\ U_{(k-1), k, l} \\ U_{(k+1), k, l} \\ \vdots \\ U_{K, k, l}\end{array}\right] \sim$

M.V.N. $\left(\mathbf{0}, \boldsymbol{\Sigma}_{k}=\left[\begin{array}{lll}\tau_{0}^{2} & \ldots & \tau_{0 K} \\ \vdots & \ddots & \vdots \\ \tau_{0 K} & \ldots & \tau_{K}^{2}\end{array}\right]\right)$. The $\gamma$ s are used to represent the fixed effects for a skill, and the $U$ s are used for the random effects of the individual learning materials. More specifically, $\gamma_{0, k, 0}$ denotes the overall log-odds that a learner with no mastered skills switches from nonmastery to mastery on skill $k$ after learning a material targeting $k$, and $\mathbf{U}_{0, k, l}$ is the incremental "approachability" of material $l$ on learning $k$, which either increases $\left(U_{0, k, l}>0\right)$ or decreases $\left(U_{0, k, l}<0\right)$ the log-odds of acquiring skill $k$. In addition, because the learning of one skill may depend on the previous mastery of another related skill, we also considered the mastery of other skills as a covariate of the learning of skill $k$. Thus, fixed and random slopes for each skill other than $k$, denoted $k^{\prime}$, were introduced. We used $\gamma_{k^{\prime}, k, 0}$ to denote the overall effect of mastery of $k^{\prime}$ on the learning of $k$, and $U_{k^{\prime}, k, l}$ was used to represent material $l$ 's additional "reliance" on skill $k^{\prime}$.

Intuitively, the fixed intercepts, $\gamma_{0, k, 0} \mathrm{~s}$, tells us the overall probability of learning a skill $k$ for learners who haven't mastered any skills, without consideration of the material's characteristics. Since different learning materials can differ in their approachability (e.g., one material may be easily understood by students without backgrounds, whereas another does not), the random intercepts, $U_{0, k, l} \mathrm{~s}$ were introduced to capture each material's approachability. The fixed slopes, $\gamma_{k^{\prime}, k, 0} \mathrm{~s}$, tell us overall, how much does the mastery of $k^{\prime}$ affect the learning outcome of $k$. While some skills may be relatively independent, others could be strongly related. For instance, it is usually perceived that the mastery of addition is a prerequisite to understanding multiplication. Therefore, the mastery of addition can have a strong influence on whether a learner could understand multiplication. Lastly, different learning materials can rely on the mastery of other skills at different degrees. As an example, we consider two video lectures on multivariate statistics, with the first one providing an introduction to linear algebra and the second one jumping directly to the matrix-based derivations of multivariate distribution means and variances. The first video would require much less previous background on linear algebra than the second. Another example is the instruction of the same skill with different approaches: We could have two instructional tools teaching the students about the "right-hand rule" in electromagnetism, with the first one teaching the students how to use their right hand to determine the direction of the magnetic force, and the second one explaining how the rule works based on vector cross products. The former would barely require any background knowledge, while the latter would require some background in linear algebra. For this reason, we introduce the random slopes for each 
specific learning material, $U_{k^{\prime}, k, l} \mathrm{~s}$, indicating to what extent a specific material $l$ would rely on prior mastery of $k^{\prime}$ in the process of learning $k$.

The proposed model differs from the model by Wang et al. (2016) mainly in terms of how the characteristics of the administered learning intervention and the learner contribute to the learning outcomes. In the hidden Markov DCM proposed by Wang et al. (2016), the learner's general ability, the list of skills s/he has mastered up to the current time, and the number of practice items s/he has completed contribute linearly to the logit of the transition probability from non-mastery to mastery. By using the count of the number of practice items as a covariate, the model assumes that different practice items on the same skill contribute equally to the transition probability. This model would be most suitable for learning programs where students learn through and are assessed after repeated practice, and where different practice problems can be regarded as equivalent (e.g., arithmetic questions where different questions only differ in the numbers and arithmetic operations they use). The proposed multilevel logistic hidden Markov model, on the other hand, contains models the main effect of the specific learning intervention and its interactions with the learner's current profile. Different learning interventions are hence assumed to vary in terms of how effective they are, both in general and for a student with a specific attribute pattern. The proposed model is thus suitable when we can assess the learner after each intervention, and when interventions targeting the same skill are assumed to differ in their effectiveness. An example would be a piece of plain text and a computer-based interactive exercise, both of which intends to teach a learner how to set the time on a clock: Although they teach the same thing, the differences in the media they use to convey the points give educators a reason to suspect their differences in effectiveness, which, under the proposed model, can be explicitly taken into account.

We treat the slope and intercept of different learning materials as random effects for several reasons: First, educators are often interested in evaluating different interventions' effectiveness. Under our model, the random intercepts can directly be used to compare different materials' overall effectiveness to students in the baseline group (i.e., $\boldsymbol{\alpha}_{i}=\mathbf{0}$ ), and to get the materials' effectiveness for other groups, the effectiveness could be calculated as the random intercept plus the random slope for that material. Second, for the multilevel model, the distribution of the random effects is modeled with a multivariate normal distribution. In the learning context, we may assume that the approachability of a material could be related to the degree of its requirement of a specific prerequisite. For example, a material deemed to be "harder" for students with none of the mastered skills may have a high requirement on another skill. Therefore, we use the covariance matrix of the learning material's random effects to capture potential relationships between different characteristics of learning materials.

Under the current model, a confirmatory approach is taken-that is, at time $t$, if the students were assigned a material targeting a specific skill, $k$, then we assume that only the mastery status on skill $k$ can change from time $t$ to time $t+1$. Here, we focus on the case of one target skill only per material. In that case, given the attribute pattern at time $t, \boldsymbol{\alpha}_{i, t}$, the probability of transitioning to pattern $\boldsymbol{\alpha}_{i, t+1}$ after receiving a learning material $l$ targeting skill $k$ is

$P\left(\boldsymbol{\alpha}_{i, t+1} \mid \boldsymbol{\alpha}_{i, t}\right)= \begin{cases}\left.\left.P\left(\alpha_{i, k, t+1}=1 \mid \cdot\right)^{\alpha_{i, k, t+1}\left[1-P\left(\alpha_{i, k, t+1}\right.\right.}=1 \mid \cdot\right)\right]^{1-\alpha_{i, k, t+1}}, & \text { if } \boldsymbol{\alpha}_{i,[k], t}=\boldsymbol{\alpha}_{i,[k], t+1}, \\ 0, & \text { otherwise. }\end{cases}$

In the equation above, $P\left(\alpha_{i, k, t+1=1 \mid}\right)=P\left(\alpha_{i, k, t+1}=\right.$ $\left.1 \mid \boldsymbol{\alpha}_{i, t}, \boldsymbol{\gamma}_{k}, \mathbf{U}_{k, l}\right)$ denotes the conditional probability of mastering a skill after learning, as given in equation (3).

The transition model in equation (4) is used to describe the changes of the students' attribute patterns across time. However, the students' latent mastery status cannot be directly observed and needs to be measured using assessment items. In our design, we assume that for each student at each time point, a set of $J_{t}$ items are administered to them to assess their mastery of skills in the curriculum. For simplicity, in the current study we used the DINA model in equation (1) for the responses. However, the modeling framework can be extended to other CDMs by swapping the DINA measurement model with other CDMs, such as the reduced Reparameterized Unified Model (rRUM; Hartz, 2002) or the generalized-DINA (G-DINA; dela Torre, 2011) model.

\section{Model estimation}

\section{Bayesian formulation}

Similar to Wang et al. (2016), a Bayesian modeling framework is used to estimate the parameters of the proposed model. Let $\boldsymbol{p}(\boldsymbol{\pi})$, where $\pi=\left[\pi_{1}, \ldots, \pi_{2^{K}}\right]$ denote the prior distribution of the population membership probabilities of each attribute pattern at the initial stage, 
$t=1$, let $p(\gamma)$ be the prior distribution for the fixed effects, and let $p(\Sigma)$ be the prior distribution for the covariance of the random effects, denoted as $\boldsymbol{\Sigma}=\left[\boldsymbol{\Sigma}_{1}, \ldots, \boldsymbol{\Sigma}_{K}\right]$, where $\boldsymbol{\Sigma}_{k}$ represents the covariance matrix of the random effects of materials for skill $k$. We further use $k_{i t}$ and $l_{i t}$ to denote the skill and material given to student $i$ at time $t$, respectively, and let $\mathbf{X}=\left[X_{1}, \ldots, X_{T}\right]$ represent the observed response data across all time points, where $X_{t}$ is the $N \times J_{t}$ response matrix of all the $N$ learners at time $t$. Then the joint likelihood of the observed responses, learning materials' random effects, latent attribute patterns, population membership probabilities, the DINA model item parameters, the learning model's fixed effects, and the covariance matrices of the random effects is

$$
\begin{aligned}
L(\mathbf{X}, \boldsymbol{\alpha}, \mathbf{U}, \boldsymbol{\pi}, \boldsymbol{\Sigma}, \boldsymbol{\gamma}, \mathbf{s}, \mathbf{g})= & \prod_{i=1}^{N}\left\{\pi_{c} P\left(\mathbf{X}_{i 1} \mid \boldsymbol{\alpha}_{i, 1}=\boldsymbol{\alpha}_{c}, \mathbf{s}, \mathbf{g}\right)\right. \\
& \left.\times \prod_{t=1}^{T-1}\left[P\left(\boldsymbol{\alpha}_{i, t+1} \mid \boldsymbol{\alpha}_{i, t}, \boldsymbol{\gamma}_{k_{i t}}, U_{k_{i t}, l_{i t}}\right) P\left(\mathbf{X}_{i, t+1} \mid \boldsymbol{\alpha}_{i, t+1}, \mathbf{s}, \mathbf{g}\right)\right]\right\} \\
& \times \prod_{k=1}^{K} \prod_{l=1}^{L_{k}}\left[P\left(\mathbf{U}_{k, l} \mid \mathbf{\Sigma}_{k}\right)\right] p(\boldsymbol{\pi}) p(\boldsymbol{\gamma}) p(\boldsymbol{\Sigma}) .
\end{aligned}
$$

Based on this formulation, a learner $i$ 's attribute pattern at the initial time point, $\boldsymbol{\alpha}_{i, 1}$, follows a multinomial distribution, i.e.,

$P\left(\boldsymbol{\alpha}_{i, 1}=\boldsymbol{\alpha}_{c}\right)=\prod_{c}^{2^{K}} \pi_{c}^{\mathcal{I}\left(\boldsymbol{\alpha}_{i, 1}=\boldsymbol{\alpha}_{c}\right)}$,

where the prior for the population membership probabilities at the initial time point is

$\pi=\left[\pi_{1}, \ldots, \pi_{2}{ }^{K}\right] \sim \operatorname{Dirichlet}\left(\boldsymbol{\delta}_{0}\right)$.

We further assign the following priors to the fixed effects,

$\gamma_{0, k, 0} \sim N\left(\mu_{*}, \sigma_{*}^{2}\right)$,

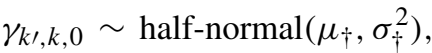

for $\forall k$ and $\forall k^{\prime} \neq k$. A half-normal prior distribution for the fixed slopes, $\gamma_{k^{\prime}, k, 0} \mathrm{~s}$, is used to restrict their support on the positive real numbers. By doing this, we impose the assumption that the existing mastery of any other skills will not decrease the probability of learning the current skill. For the random effects $\mathbf{U}_{k, l} \sim \operatorname{M.V.N.}\left(\mathbf{0}, \boldsymbol{\Sigma}_{k}\right)$, we assume for all $k$, the prior for the covariance is given by the Inverse-Wishart distribution, where

$\boldsymbol{\Sigma}_{k}^{-1} \sim \operatorname{Wishart}(\mathbf{S}, v)$,

with $\mathbf{S}$ being the scale matrix and $v$ the degrees of freedom.

Lastly, adopting the methods in Culpepper (2015), we assign a truncated Beta prior distribution to the DINA model item parameters, i.e.

$p\left(s_{j}, g_{j}\right) \propto s_{j}^{a_{s}-1}\left(1-s_{j}\right)^{b_{s}-1} g_{j}^{a_{g}-1}\left(1-g_{j}\right)^{b_{g}-1} \mathcal{I}\left(0 \leq g_{j}<1-s_{j} \leq 1\right)$.

Then, the full conditional distributions of the parameters, given the observed responses at all time points, are as follows:

- For $\boldsymbol{\alpha}_{i, t}$ : Similar to Wang et al. (2016) and Chen et al. (2017), we use a forward-backward algorithm to sequentially update the attribute patterns of each subject at each time point. Specifically,

$P\left(\boldsymbol{\alpha}_{i, t}=\boldsymbol{\alpha}_{c}\right) \propto \begin{cases}\pi_{c} P\left(X_{i 1} \mid \boldsymbol{\alpha}_{i, 1}=\boldsymbol{\alpha}_{c}\right) P\left(\boldsymbol{\alpha}_{i, 2} \mid \boldsymbol{\alpha}_{i, 1}=\boldsymbol{\alpha}_{c}, \cdot\right), & t=1 ; \\ P\left(\boldsymbol{\alpha}_{i}=\boldsymbol{\alpha}_{c} \mid \boldsymbol{\alpha}_{i, t-1}, \cdot\right) P\left(X_{i t} \mid \boldsymbol{\alpha}_{i, t}=\boldsymbol{\alpha}_{c}\right) P\left(\boldsymbol{\alpha}_{i, t+1} \mid \boldsymbol{\alpha}_{i, t}=\boldsymbol{\alpha}_{c}, \cdot\right), & 1<t<T ; \\ P\left(X_{i T} \mid \boldsymbol{\alpha}_{i, T}=\boldsymbol{\alpha}_{c}\right) P\left(\boldsymbol{\alpha}_{i, T}=\boldsymbol{\alpha}_{c} \mid \boldsymbol{\alpha}_{i, T-1}, \cdot\right), & t=T,\end{cases}$

where $P\left(\boldsymbol{\alpha}_{i, t+1}=\boldsymbol{\alpha}_{c} \mid \boldsymbol{\alpha}_{i, t}, \cdot\right)$ represents $P\left(\boldsymbol{\alpha}_{i, t+1}=\right.$ $\left.\boldsymbol{\alpha}_{c} \mid \boldsymbol{\alpha}_{i, t}, \boldsymbol{\gamma}_{k_{i, t}}, \mathbf{U}_{k_{i, t}, l_{i, t}}\right)$ and is given in equations (3) and (4).

- For population proportions of the attribute patterns at time 1 , the full conditional distribution of $\pi$ is still a Dirichlet distribution, with

$\pi \mid \boldsymbol{\alpha}_{1,1} \ldots, \boldsymbol{\alpha}_{N, 1} \sim \operatorname{Dirichlet}\left(\delta_{0}+\tilde{N}\right)$, where $\tilde{N}=\left[\sum_{i=1}^{N} \mathcal{I}\left(\boldsymbol{\alpha}_{i 1}=\boldsymbol{\alpha}_{1}\right), \ldots, \sum_{i=1}^{N} \mathcal{I}\left(\boldsymbol{\alpha}_{i 1}=\right.\right.$ $\left.\left.\boldsymbol{\alpha}_{2^{K}}\right)\right]$

- For fixed effect $\boldsymbol{\gamma}_{k}$, we have

$$
P\left(\boldsymbol{\gamma}_{k} \mid \boldsymbol{\alpha}, \mathbf{U}_{k, .}\right) \propto p\left(\boldsymbol{\gamma}_{k}\right) \prod_{t=1}^{K} \prod_{i: k_{i, t}=k} P\left(\boldsymbol{\alpha}_{i, t+1} \mid \boldsymbol{\alpha}_{i, t}, \boldsymbol{\gamma}_{k}, \mathbf{U}_{k, l_{i, t}}\right)
$$


- For random effects $\mathbf{U}_{k, l}$, we have

$$
\begin{aligned}
& P\left(\mathbf{U}_{k, l} \mid \boldsymbol{\Sigma}_{k}, \boldsymbol{\alpha}, \boldsymbol{\gamma}_{k}\right) \propto \prod_{t=1}^{K} \prod_{i: k_{i, t}=k} \&_{l_{i, t}=l} \\
& P\left(\boldsymbol{\alpha}_{i, t+1} \mid \boldsymbol{\alpha}_{i, t}, \boldsymbol{\gamma}_{k}, \mathbf{U}_{k, l}\right) P\left(\mathbf{U}_{k, l} \mid \mathbf{\Sigma}_{k}\right) .
\end{aligned}
$$

- For the covariance matrices of random effects, $\boldsymbol{\Sigma}_{k}$, conditioning on the random effects of materials targeting skill $k, \boldsymbol{\Sigma}_{k}$ follows an Inverse-Wishart distribution, i.e.,

$\boldsymbol{\Sigma}_{k}^{-1} \mid \mathbf{U}_{1, k}, \ldots, \mathbf{U}_{L_{k}, k} \sim \operatorname{Wishart}\left(\mathbf{S}^{*}, v^{*}\right)$,

where $\mathbf{S}^{*}=\mathbf{U}^{T} \mathbf{U}+\mathbf{S}$, and $v^{*}=L_{k}+v$, and $\mathbf{U}$ represents the matrix of the random effects corresponding to skill $k$, with $\mathbf{U}_{k, l}$ as the $l$ th row.

- For the DINA model parameters, $\mathbf{s}$ and $\mathbf{g}$, the posterior distribution, given the responses and attribute patterns, is a truncated beta distribution, with

$p\left(s_{j}, g_{j} \mid \boldsymbol{\alpha}, \mathbf{X}\right) \propto s_{j}^{a_{s j}-1}\left(1-s_{j}\right)^{b_{s j}-1} g_{j}^{\left(a_{g j}-1\right)}\left(1-g_{j}\right)^{b_{g j}-1} \mathcal{I}\left(0 \leq g_{j}<\left(1-s_{j}\right) \leq 1\right)$,

where

$$
\begin{aligned}
& a_{s j}=\sum_{i: X_{i j}=0} \eta_{i j}+a_{s}, \quad b_{s j}=\sum_{i: X_{i j}=1} \eta_{i j}+b_{s}, \\
& a_{g j}=\sum_{i: X_{i j}=1}\left(1-\eta_{i j}\right)+a_{g}, b_{g j}=\sum_{i: X_{i j}=0}\left(1-\eta_{i j}\right)+b_{g} .
\end{aligned}
$$

\section{Parameter estimation}

A Metropolis-Hastings within Gibbs algorithm is used to estimate the parameters of the proposed model. Let $\boldsymbol{\alpha}_{i, t}^{0}, \mathbf{U}^{0}, \boldsymbol{\gamma}^{0}, \boldsymbol{\pi}^{0}, \mathbf{s}^{0}, \mathbf{g}^{0}$, and $\boldsymbol{\Sigma}^{0}$ denote the initial values of our parameters of interest, the following procedures could be used to sample from the posterior distribution of the parameters. At each iteration of the MCMC chain:

(1) For each $i=1,2, \ldots, N, t=1,2, \ldots, T$, sample $\boldsymbol{\alpha}_{i, t}^{r}$, given $\mathbf{U}^{r-1}, \boldsymbol{\gamma}^{r-1}, \boldsymbol{\pi}^{r-1}$, and $\mathbf{s}^{r-1}, \mathbf{g}^{r-1}$;

(2) Sample $\boldsymbol{\pi}^{r}$ based on $\boldsymbol{\alpha}_{1,1}^{r}, \ldots, \boldsymbol{\alpha}_{N, 1}^{r}$;

(3) For each $\gamma_{k^{\prime}, k, 0}$, where $k, k^{\prime}=0,1, \ldots, K$, sample $\gamma_{k^{\prime}, k, 0}^{r}$ from the uniform distribution in an interval around $\gamma_{k^{\prime}, k, 0}^{r-1}$, and accept with probability $\frac{P\left(\gamma_{k^{\prime}, k, 0}^{r} \mid \boldsymbol{\alpha}_{t+1}^{r}, \boldsymbol{\alpha}_{t}^{r}, \mathbf{U}_{k, \cdot}^{r-1}\right)}{P\left(\gamma_{k^{\prime}, k, 0}^{r-1} \mid \boldsymbol{\alpha}_{t+1}^{r}, \boldsymbol{\alpha}_{t}^{r}, \mathbf{U}_{k, \cdot}^{r-1}\right)} ;$

(4) For each $k=1,2, \ldots, K, l=1,2, \ldots, L_{k}$, sample $\mathbf{U}_{k, l}^{r}$ from the Uniform distribution of a small interval around $\mathbf{U}_{k, l}^{r-1}$, and accept with probability $\frac{P\left(\mathbf{U}_{k, l}^{r} \mid \mathbf{\Sigma}_{k}^{r-1}, \boldsymbol{\alpha}_{t+1}^{r}, \boldsymbol{\alpha}_{t}^{r}, \boldsymbol{\gamma}_{k}^{r}\right)}{P\left(\mathbf{U}_{k, l}^{r-1} \mid \mathbf{\Sigma}_{k}^{r-1}, \boldsymbol{\alpha}_{t+1}^{r}, \boldsymbol{\alpha}_{t}^{r}, \boldsymbol{\gamma}_{k}^{r}\right)}$.

(5) For each $k=1,2, \ldots, K$, sample $\Sigma_{k}^{r}$ from the inverse-Wishart distribution updated based on $\mathbf{U}_{k, 1}^{r}, \ldots, \mathbf{U}_{k, L_{k}}^{r}$.

(6) For each item $j$, sample $s_{j}, g_{j}$ from the truncated beta distribution, given $\boldsymbol{\alpha}^{r}$ and $\mathbf{X}$.

\section{Model identification}

We note that for the logistic transition model given in equation (3), for each of the slopes and intercepts, $\lambda_{k^{\prime}, k, l}$ and $\lambda_{0, k, l}$ remain the same if we add a constant to the corresponding fixed effect and subtract the constant from each of the corresponding random effects. In other words,

$$
\begin{aligned}
& \lambda_{0, k, l}=\left(\gamma_{0, k, 0}+c\right)+\left(U_{0, k, l}-c\right), \\
& \lambda_{k^{\prime}, k, l}=\left(\gamma_{k^{\prime}, k, 0}+c\right)+\left(U_{k^{\prime}, k, l}-c\right) .
\end{aligned}
$$

Although we are unable to exactly fix the location of the fixed and random effects, they can be softly centered by imposing a mean of $\mathbf{0}$ to the multivariate normal distribution of the random effects (e.g., Gelman, Carlin, Stern, Dunson, Vehtari, \& Rubin, 2014).

\section{Simulation study}

\section{True parameter generation}

We conduct a series of simulation studies to evaluate the recovery of model parameters using the proposed algorithm. The design of the learning process is as follows: The curriculum is assumed to contain $K=4$ skills. At the initial time point, $t=1$, all students are administered $J_{t}=15$ items. Following the assessment, each student is administered a random learning material targeting a random skill. Specifically, for student $i$ at time $t$, we randomly select one of the 4 skills as the target skill $\left(k_{i, t}\right)$, and among all materials available for learning the target skill, we randomly select one $\left(l_{i, t}\right)$ as the material to be administered to student $i$ at time $t$. Following the learning of the material, students are assessed again with 15 items, and this process iterates up to the final time point, $T=5$. Figure 1 provides an illustration of the learning program design used in the simulation studies.

Because our main interest is the recovery of the parameters in the learning model, two factors are considered, including the number of students who receive each specific training material $\left(N_{l, k}=50\right.$ and 100), and the number of 


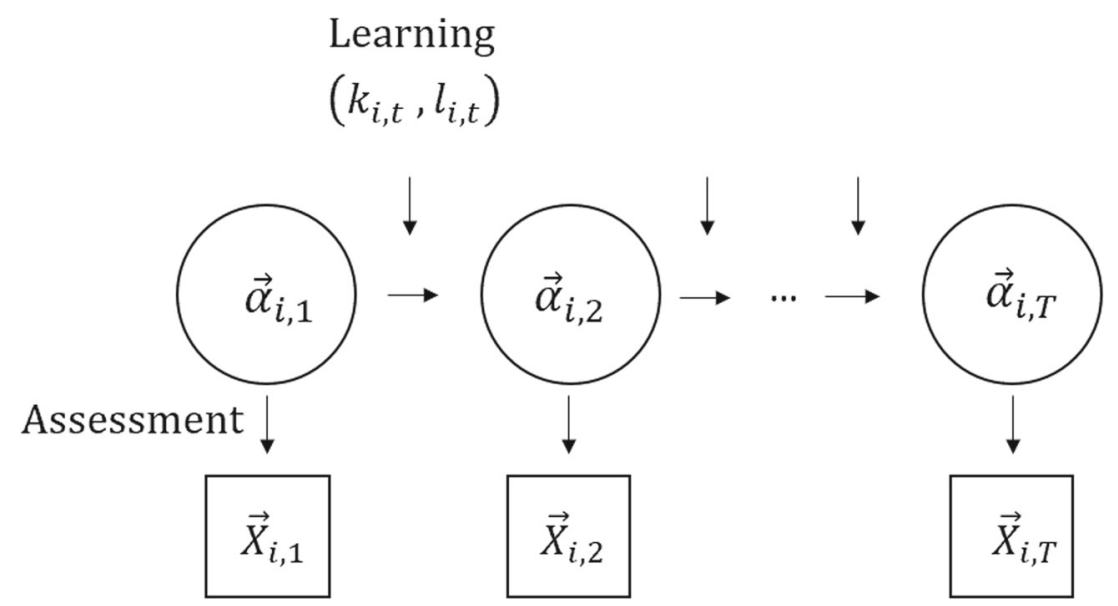

Fig. 1 Illustration of the learning design used in the simulation studies. Following the learning of skill $k_{i, t}$ and material $l_{i, t}$, a student's skill pattern changes from $\boldsymbol{\alpha}_{i, t}$ to $\boldsymbol{\alpha}_{i, t+1}$. The student's current skill mastery, $\boldsymbol{\alpha}_{i, t}$ is observed through the responses to assessment items, $\mathbf{X}_{i, t}$

materials available for each skill $\left(L_{k}=10,30\right.$, and 50). The $L_{k}$ s under each condition are chosen to mimic the number of available online resources for each Common Core Math standard on the OpenEd repository. Based on these two factors, we can calculate the total number of subjects in each condition. For example, in the case of 50 students per material and ten materials per skill, the total sample size would be $N=50 \times 10 \times K=50 \times 10 \times 4=2000$. Such large samples are very difficult to collect in a classroom learning environment. However, they are achievable in online learning environments, where students from different backgrounds can access the learning materials anytime and anywhere. Ten repetitions of the simulation were performed under each condition.

The initial attribute patterns of the students, $\boldsymbol{\alpha} .1$, are generated following similar methods as in Chiu and Köhn (2016). Specifically, for each learner $i$, we first generate a $K$-dimensional multivariate normal variable $\boldsymbol{Z}_{i} \sim$ M.V.N. $\left(\mathbf{0}, \boldsymbol{\Sigma}_{Z}\right)$, where the diagonal entries of $\boldsymbol{\Sigma}_{Z}$ are 1, and the off-diagonal entries are .2 . That is, we assume that at the initial time point, the higher-order continuous traits behind each skill are correlated at $\rho=.2$. Next, $\alpha_{i, k, 1}=$ $\mathcal{I}\left(Z_{i k} \geq \Phi^{-1}\left(\frac{k}{K+1}\right)\right)$ was obtained for each $k$, giving the true attribute pattern of subject $i$ at the initial time point, $\boldsymbol{\alpha}_{i, t}$.

The true values of the fixed intercepts, $\gamma_{0, k, 0} \mathrm{~s}$, were generated from $N(-1,1)$, and the fixed slopes, $\gamma_{k^{\prime}, k, 0} \mathrm{~s}$ were sampled from Uniform $(0,2)$. The covariance matrices of the random effects of each skill, $\boldsymbol{\Sigma}_{k}, k \in\{1, \ldots, K\}$, were assumed to have diagonal entries of .3 and off diagonal entries of .1. Based on the covariance matrices, the true random intercepts and slopes were generated from the multivariate normal distribution, with $\mathbf{U}_{k, l} \sim$
M.V.N. $\left(\mathbf{0}, \boldsymbol{\Sigma}_{k}\right)$. Based on the random and fixed effects of the learning model, the learners' subsequent $\boldsymbol{\alpha}$ s can be generated based on transition probabilities as defined in equations (3) and (4).

Five blocks of DINA model items, with $J_{t}=15$ items per block, were simulated. The true slipping and guessing parameters were generated from $U(.15, .3) . \mathrm{Gu}$ and $\mathrm{Xu}$ (2018) provided the sufficient and necessary conditions for DINA model parameter identifiability in static assessments. Specifically, the item parameters $\mathbf{s}, \mathbf{g}$ and the population membership probabilities $\pi$ are identifiable if each skill is assessed with at least three items, and after row permutations, the Q-matrix can be written as $\left(\frac{\mathcal{I}_{K}}{\mathcal{Q}^{*}}\right)$, where $\mathcal{I}_{K}$ is a $K \times K$ identity matrix, and the columns of $\mathcal{Q}^{*}$ are distinct. In our simulation studies, for each block of items, the Q-matrices were generated to include at least three items that exclusively measure the $k$ th skill for all $k$. We could see that in this case, the Q-matrices will satisfy the identifiability conditions in $\mathrm{Gu}$ and $\mathrm{Xu}$ (2018). All students are assumed to receive item block $t$ at time $t$. Because we assumed the learning process to be monotonic, the proportion of students mastering a large number of skills are expected to increase as the learning process continues. In that case, the proportion of students mastering few skills may be small at later time points. Thus in the present study, we assumed that the DINA item parameters are known a priori. The simultaneous estimation of the DINA model parameters and the rest of the model parameters could be easily achieved by adding an additional step of updating the $\mathbf{s}$ and gs. Future studies could consider randomizing the order the items for each examinee, so that each item could have sufficient sample size for the estimation of its 
parameters. Based on the students' attribute patterns at each time point, the block of items they are administered at time $t$, and the Q-matrix and parameters of the items in each block, the subjects' responses can be randomly generated based on the DINA model item response function.

\section{Parameter estimation}

The proposed MCMC algorithm was used to estimate the model parameters. The following prior distributions were used for the model parameters:

$\boldsymbol{\pi} \sim \operatorname{Dirichlet}(\mathbf{1}), \gamma_{0, k, 0} \sim N(0,1), \quad \gamma_{k^{\prime}, k, 0} \sim \operatorname{half-normal}\left(0,3^{2}\right), \quad \boldsymbol{\Sigma}_{k}^{-1} \sim \operatorname{Wishart}\left(\mathcal{I}_{K}, 8\right)$.

These prior parameters were selected either as uninformative or to reflect how we expect the model parameters to be distributed under the proposed model. Specifically, without prior knowledge, the prior distribution of initial attribute pattern probabilities is assumed to be uniform, and the random slopes for different skills are assumed to be independent. We chose $N(0,1)$ as the prior for the fixed intercepts because without prior knowledge, we assume that when students were assigned to learn a specific skill, there is about $50 \%$ chance to switch from non-mastery to mastery. And a half-normal $\left(0,3^{2}\right)$ prior distribution for the fixed slopes was selected because without prior knowledge, we assume the learning of one skill is likely unrelated to the mastery of another, and, given that the fixed intercepts are mostly distributed between -3 and 3 under their prior distributions, a fixed slope above 10 will not have meaningful interpretations in the logistic transition model.

We assigned random initial values to the model parameters to start the MCMC algorithm. Specifically, the initial population membership probabilities, $\pi$, were generated from the Dirichlet distribution with $\delta=\mathbf{1}$; the covariance matrices of the random effects, $\boldsymbol{\Sigma}_{k} \mathrm{~s}$, were set to be the identity matrix; initial values for $\mathbf{U}$ were randomly sampled from the multivariate normal distribution with mean $\mathbf{0}$ and covariance equal to the initial $\boldsymbol{\Sigma}_{k}$, the initial fixed slopes were randomly generated from $U(0,2)$, and the initial fixed intercepts were randomly sampled from $N(-1,1)$; lastly, the initial attribute patterns of the subjects at $t=1$ were randomly sampled based on the initial value of $\pi$, and the subsequent $\alpha$ s for $t=2, \ldots, 5$ were generated based on the learning model and the initial learning model parameters. Then, we implemented the MH within Gibbs algorithm to iteratively update the parameters. For the Metropolis-Hastings sampling of the fixed and random effects, window sizes of the uniform distributions around the old values were selected so that the average acceptance rate for $\boldsymbol{\gamma}$ and for $\mathbf{U}$ were both between $20 \%-40 \%$. A chain length of 20,000 iterations was used for each condition, with burn-in of 10,000 iterations. The MCMC algorithms were written in $\mathrm{C}++$ and implemented in $\mathrm{R}$ through the Rcpp (Eddelbuettel et al., 2011) package. The complete code for the MCMC algorithm is provided in the Appendix.

\section{Evaluation criteria}

The proposed model and estimation methods are evaluated in terms of two aspects, parameter convergence and parameter recovery. To evaluate the parameter convergence, we randomly generated one set of response data under the $L_{k}=10, N_{k, l}=50$ condition, and with different starting values, we separately ran the MCMC algorithm on this data set with five chains, each with 30,000 iterations. The convergence of the parameters was evaluated by monitoring the change in the maximum Gelman-Rubin proportional scale reduction factor (PSRF; Gelman \& Rubin, 1992) across all continuous model parameters: At different chain lengths ranging from 200 to 30,000, we use the first halves of the MCMC chains as the burn-in cycles and use the second halves to compute the PSRF. By conventions, a PSRF below 1.2 provides evidence for parameter convergence.

Our second objective is to evaluate the recovery of the true parameters with the proposed algorithms. Based on the parameter samples from the MCMC, we can calculate the expected a posteriori (EAP) estimates of the $\mathbf{U}, \boldsymbol{\gamma}, \boldsymbol{\Sigma}$, and $\boldsymbol{\alpha}$ using

$\hat{\theta}_{E A P}=\frac{\sum_{r=T_{b u r n}+1}^{T_{t o t}} \theta^{r}}{T_{t o t}-T_{b u r n}}$

where $\theta^{r}$ denotes the parameter sample from the $r$ th iteration, and $T_{\text {tot }}$ and $T_{\text {burn }}$ are the total length and burnin length of the MCMC chains, respectively. For the $\alpha \mathrm{s}$, because for each $\alpha_{i, k, t}$, the EAP will give a value between 0 and 1 , we further dichotomize each $\alpha_{i, k, t}$ and set it to 1 if the corresponding EAP is greater than .5, and 0 otherwise.

The estimation accuracy of the attribute patterns at each time point is evaluated by the attribute-wise agreement rate (AAR), where

$A A R\left(\hat{\boldsymbol{\alpha}}_{t}\right)=\frac{\sum_{i=1}^{N} \sum_{k=1}^{K} \mathcal{I}\left(\alpha_{i, k, t}=\hat{\alpha}_{i, k, t}\right)}{N \times K}$, 
and the estimation accuracy of $\boldsymbol{\pi}$ and $\boldsymbol{\Sigma}$ is evaluated based on their bias and RMSE, given by

$$
\operatorname{Bias}(\hat{\theta})=\hat{\theta}_{E A P}-\theta, \quad \operatorname{RMSE}(\hat{\theta})=\sqrt{\frac{\sum_{r=T_{\text {burn }}+1}^{T_{\text {tot }}}\left(\theta^{r}-\theta\right)^{2}}{T_{\text {tot }}-T_{\text {burn }}}},
$$

where $\theta$ denotes the true value of the parameter.

Because we used soft centering for the fixed and random effects, the estimated values of both might be slightly shifted from the true values. However, if the algorithm could recover the parameters accurately, the composite of the estimated fixed and random effects, $\lambda \mathrm{s}$, should be close to true values. We hence cannot use bias as a criteria for the recovery of the fixed and random effects. Instead, the Pearson correlations between the estimated and the true fixed and random effects are computed.

\section{Results}

Parameter convergence Figure 2 presents the progression of the Gelman-Rubin PSRF statistic as a function of chain length. As the plot suggests, the MCMC chains have quickly
Table 1 Attribute-wise agreement rates (AARs) at different time points under different simulation conditions

\begin{tabular}{lllllll}
\hline$L_{k}$ & $N_{l, k}$ & $t=1$ & $t=2$ & $t=3$ & $t=4$ & $t=5$ \\
\hline 10 & 50 & .884 & .835 & .836 & .844 & .839 \\
10 & 100 & .891 & .839 & .841 & .844 & .834 \\
30 & 50 & .887 & .847 & .844 & .839 & .829 \\
30 & 100 & .882 & .838 & .836 & .839 & .841 \\
50 & 50 & .892 & .839 & .840 & .837 & .830 \\
50 & 100 & .891 & .835 & .837 & .838 & .836 \\
\hline
\end{tabular}

$L_{k}$ stands for the number of available materials targeting skill $k$, and $N_{l, k}$ stands for the number of learners who are administered each material. Results are aggregated across ten repetitions

stabilized after less than 3000 iterations, with the maximum PSRF across all continuous parameters falling below 1.2 This suggests that the parameter samples have stabilized with a relatively small number of iterations.

True parameter recovery Table 1 presents the accuracy of attribute estimates at different time points in the learning process, averaged across ten repetitions under each

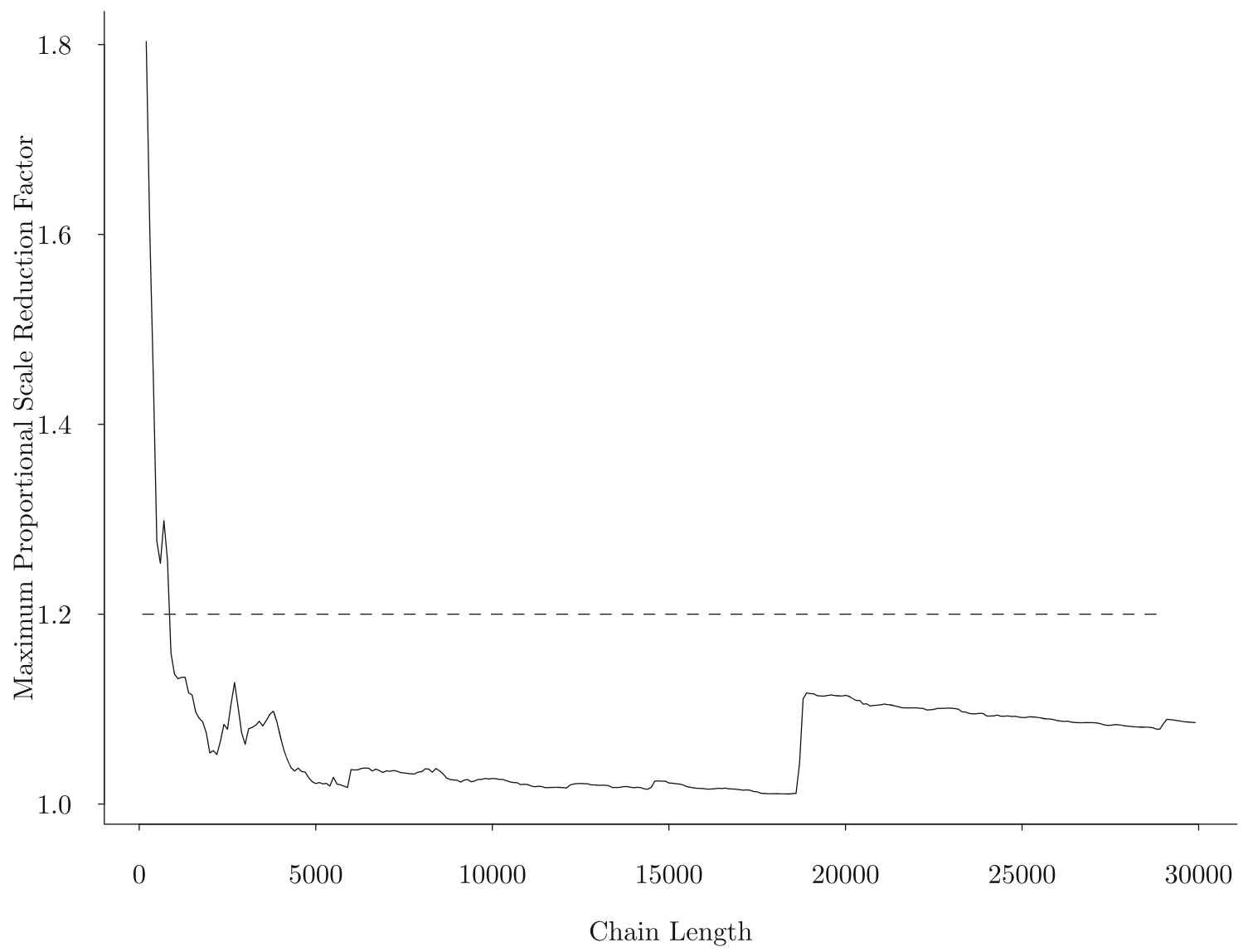

Fig. 2 The progression of the maximum Gelman-Rubin PSRF across all parameters as chain length increases. The dashed line indicates the threshold of $P S R F=1.2$ 
simulation condition. Across all conditions and all time points, the attribute-wise agreement rate between the true and estimated $\boldsymbol{\alpha}$ s remained above .8, indicating relatively accurate estimates of the learners' patterns at all time points. We observed that across all conditions, the AAR in the initial time point, $t=1$, was higher than that of the later time points. In addition, the AARs seemed to be higher for conditions with more learners per material. This suggests that part of the estimation error in the attribute patterns can be attributed to the errors in the random effect estimates for the transition model. For the initial time point, where the transition model takes a smaller weight in the estimation algorithm and for conditions with more data for the estimation of the transition model parameters, the attribute patterns were estimated slightly better.

We further investigated the parameter recovery of the fixed effects, $\boldsymbol{\gamma}$, and the random effects $\mathbf{U}$ for the learning materials. Figures 3 and 4 present the scatter plots of the true and estimated fixed and random effects under different simulation conditions. We combined the parameters from different repetitions into one plot for each condition. Across all conditions, the fixed effects seemed to be accurately recovered with the estimation algorithm, with the points on the scatterplot nearly forming a straight line. With increasing $N_{l, k}$ (number of students using each learning material) and $L_{k}$ (number of materials available for each skill), we see slight improvements in estimation accuracy for the fixed effects. The random effects corresponding to the learning materials demonstrated larger error. From Fig. 4, we can see that although there is strong correlation between the true and estimated random parameters, there are more deviations of the points from a straight line compared to the plot of $\boldsymbol{\gamma}$. Similar to the fixed effects, for conditions with larger $N_{l, k}$ and larger $L_{k}$, the correlation between true and estimated $U$ s appeared stronger.

Table 2 provides the Pearson correlations between true and estimated random $(\mathbf{U})$ and fixed $(\boldsymbol{\gamma})$ effects, as well as the bias and/or RMSE of the initial population membership probabilities $(\boldsymbol{\pi})$ and the covariance matrices of learning materials' random effects $(\boldsymbol{\Sigma})$ under different conditions. We omitted the bias of $\pi$ here, because the total probability of different classes sums to 1 , so the bias would always be 0 . In addition, we separately calculated the bias and RMSE of the diagonal entries $\left(\tau_{k}^{2} \mathrm{~s}\right)$ and the off-diagonal entries $\left(\tau_{k^{\prime} k}\right)$ of the covariance matrix of random effects, because the interpretations of the diagonal and off diagonal elements are different. Taking a coarse look at Table 2, we observe that the correlation between true and estimated fixed effects, $\gamma \mathrm{s}$, were above .94 across all conditions and increased slightly with larger $N_{l, k}$ and $L_{k}$, which is consistent with Fig. 3. The correlations for the random slopes and intercepts, $U \mathrm{~s}$, were in the .59 to .70 range across conditions and were higher when the number of learning materials available for each skill increased, or when the number of subjects using each material increased. One explanation is that, by increasing the number of available training materials, the distribution of the random effects could be estimated better, hence improving the random effect estimates. And when the number of subjects using a particular material is large, we have more data on the transitions of the subjects on this skill using this particular material, hence improving the random effect estimates corresponding to the material. Across all conditions, the RMSE for $\pi$ was low, but conditions with more learning materials or larger sample size per material had slightly better $\pi$ estimates. We think this is mainly due to larger overall sample sizes in those conditions. For the covariance matrices of random effects, we observe that both the variances on the diagonal and the covariances on the off-diagonal were estimated much better for 30 or 50 materials per skill than with only ten available materials per skill, as indicated by the smaller bias and RMSE values. This suggests that with 10 observations, the distribution of the random effects cannot be recovered very well. However, with 30 or 50 random observations, the true covariance matrix can be accurately recovered. Although the number of learners per material did not seem to have too large an effect on the bias of the covariance matrix entry estimates, the RMSE of the covariance matrix estimates were consistently lower when $N_{l, k}$ was large, indicating that the estimates were more stable when sample size for each material was larger.

Finally, under the condition with $K=4, L_{k}=10$, and $N_{l, k}=50$ (i.e., total sample size of 2000), running 20,000 iterations of the proposed MCMC algorithm takes approximately 1500 seconds on a Linux cluster with one core and an Intel E5-2670 processor. The computational time increases linearly with the number of materials per skill $\left(L_{k}\right)$ and the number of students taking each material $\left(N_{l, k}\right)$.

\section{Discussion}

In the present study, we proposed a hidden Markov model with both learner characteristics (i.e., previous skill pattern) and learning material characteristics (i.e., approachability and reliance on other skills) as covariates affecting the outcome of learning. By modeling the learning materials' characteristics as random effects, we could capture the underlying distribution structure of the materials' approachability and dependency on other skills, and the random slope and intercept estimates obtained for each learning material could also be used to assess their effectiveness on students with various attribute profiles. Under the proposed Bayesian estimation algorithm, model parameters of the multilevel logistic HMM were relatively accurately recovered, especially when there is a sufficient 

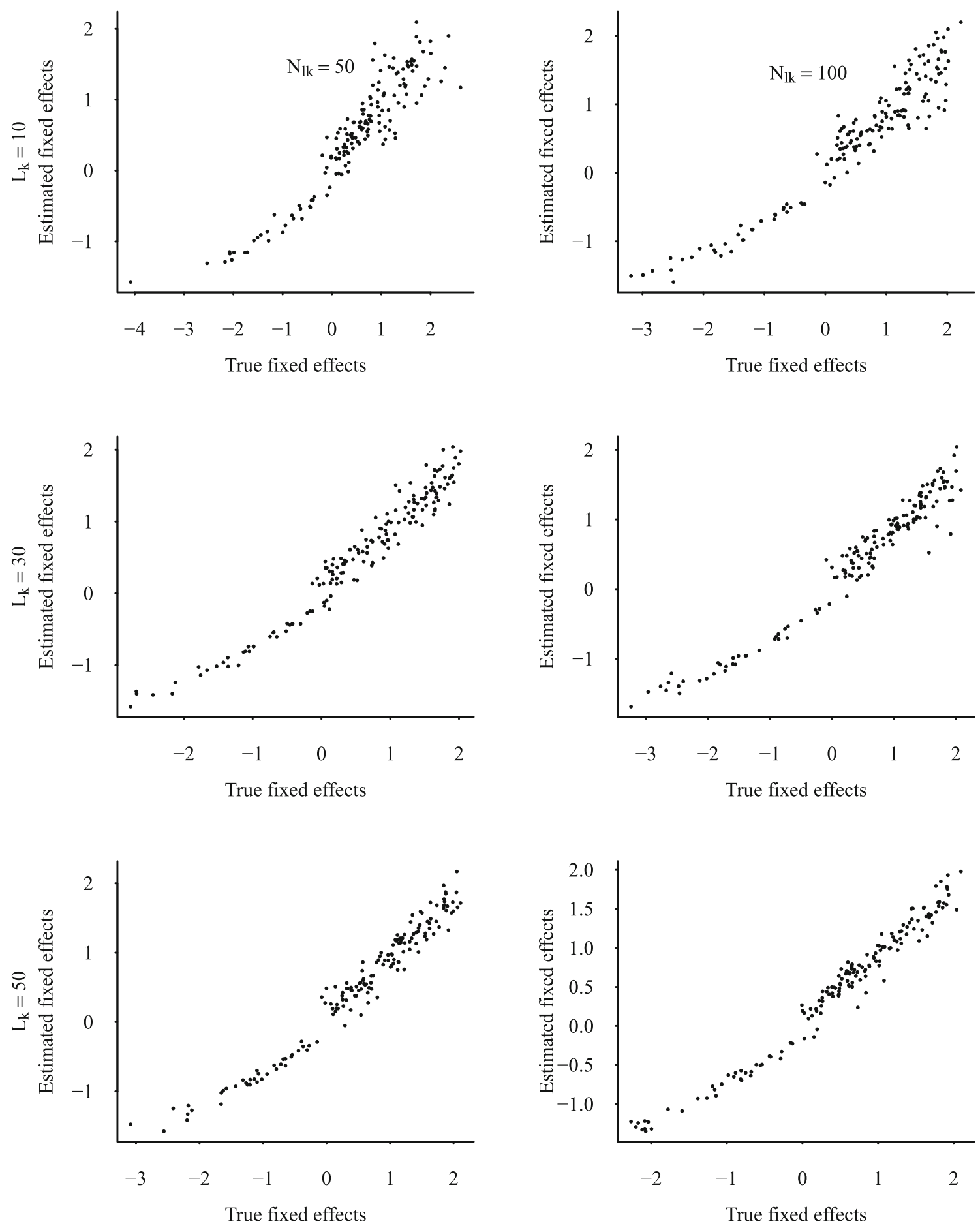

Fig. 3 Scatterplot for the true and estimated fixed effects $(\gamma)$ under different simulation conditions

number of learning materials per skill and when there is sufficient learners using each material.

There are a few potential implications of the current model. First of all, a long-term interest of educational practitioners is understanding the structural relationship between different attributes. By knowing which skills are prerequisites to others, educators can design the courses so that basic contents precede the advanced skills. In addition, if a student is assigned to learn a complex skill and fails to learn it after many trials, one possible explanation of the difficulty in learning the skill is missing prerequisites. Knowing which skills are prerequisites to an advanced, hard-to-learn skill may help educators identify why a student cannot learn effectively, and the student can 

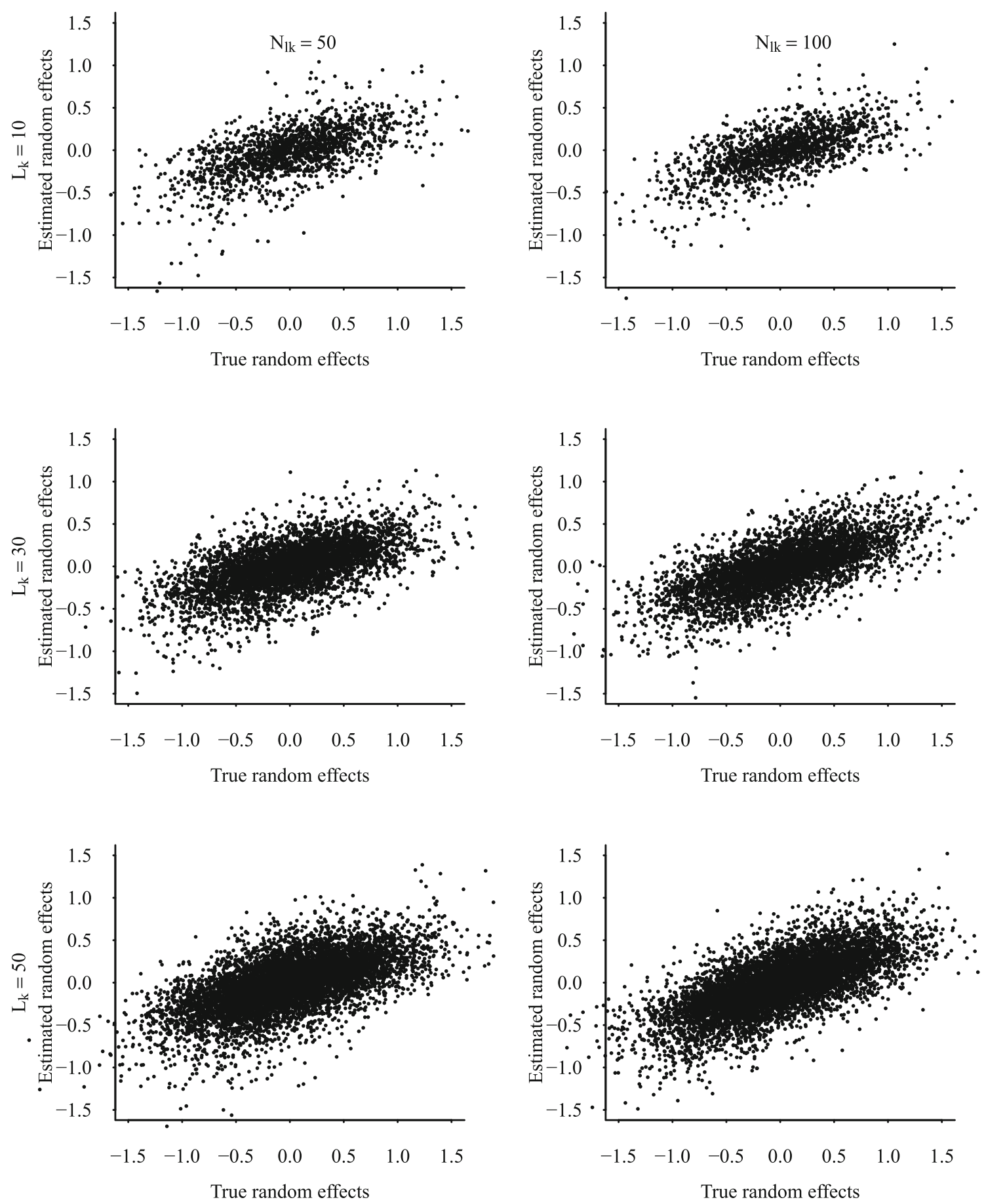

Fig. 4 Scatterplot for the true ad estimated random effects (U) under different simulation conditions

be routed back to learn the basic prerequisite. Previous research on the hierarchical structure of attributes (e.g., Leighton, Gierl, \& Hunka, 2004) usually requires the attribute hierarchies to be pre-specified by content experts. Under the current model, the dependencies between skills can potentially be inferred from the fixed slope estimates in the transition model. In other words, if the fixed slope of skill $k^{\prime}$ is large in the logistic transition model for learning skill $k$, we can infer that whether a student can learn skill $k$ successfully strongly depends on the previous mastery of skill $k^{\prime}$.

A second potential application of the proposed model is the adaptive recommendation of contents to students. In adaptive learning systems, students are sequentially assigned contents to learn. To help learners achieve their learning goals (e.g., mastering all skills) as efficiently as 
Table 2 Parameter recovery of fixed $(\gamma \mathrm{s})$ and random $(U \mathrm{~s})$ effects, initial population membership probabilities $(\boldsymbol{\pi})$, and covariance matrix for random effects $(\boldsymbol{\Sigma})$

\begin{tabular}{lllllllll}
\hline$L_{k}$ & $N_{k l}$ & $\rho_{\gamma}$ & $\rho_{U}$ & $\operatorname{RMSE}(\pi)$ & $\operatorname{Bias}\left(\tau_{k}^{2}\right)$ & $\operatorname{RMSE}\left(\tau_{k}^{2}\right)$ & $\operatorname{Bias}\left(\tau_{k^{\prime} k}\right)$ & $\operatorname{RMSE}\left(\tau_{k^{\prime} k}\right)$ \\
\hline 10 & 50 & 0.933 & 0.602 & 0.010 & 0.017 & 0.246 & 0.063 & 0.165 \\
10 & 100 & 0.961 & 0.667 & 0.008 & 0.020 & 0.198 & 0.076 & 0.063 \\
30 & 50 & 0.975 & 0.595 & 0.007 & 0.016 & 0.186 & 0.068 & 0.124 \\
30 & 100 & 0.973 & 0.678 & 0.007 & 0.018 & 0.166 & 0.107 & 0.115 \\
50 & 50 & 0.983 & 0.617 & 0.007 & 0.013 & 0.154 & 0.063 & 0.095 \\
50 & 100 & 0.985 & 0.703 & 0.006 & 0.016 & & \\
\hline
\end{tabular}

possible, content at each time point can be adaptively selected based on previous knowledge about the student's characteristics, such as his or her previous mastery on the skills in the curriculum, level of engagement in the content, or learning style (Oxman \& Wong, 2014). The current modeling framework could help us determine which materials could be deemed as "optimal" for a student: Under the current model, based on a learner's current attribute pattern estimate and the parameters of different learning materials, the proposed model can answer simple questions such as "What's the probability of mastering skill $k$ if we decide to let the student learn $k$ next and administer material $l$ to him/her?" or "Among all materials targeting skill $k$, which one will be most effective to the student right now?". It can also be used to answer more complex questions, such as "Among all possible trajectories of instructions, which one can give us the highest expected attribute pattern after $t$ stages?"

The proposed modeling framework could also be adapted to evaluate other types of interventions, such as treatments for psychological disorders. Taking the example of substance abuse, many different types of therapies exist for the treatment of alcoholism, including various types of behavioral therapies, medications, and support groups. These treatments vary in their effectiveness, and some treatments may be particularly useful for patients exhibiting specific DSM-defined symptoms. Several cognitive diagnosis models have been proposed by previous researchers for assessing the existence of symptoms in psychological disorders (e.g., Templin \& Henson, 2006, dela Torre, van der Ark, \& Rossi, 2017). These models could be used in conjunction with the proposed longitudinal model to track the progression of symptoms of patients over multiple waves. Different from the learning context, the attribute trajectory of symptoms are expected to decrease over time (i.e., from 1 to 0$)$ if the treatments are effective. The treatments can also be evaluated and compared through the random effects in the logistic transition model, which captures the probability of transitioning from 1 (possessing a symptom) to 0 (not possessing a symptom) given a specific treatment. This information could be used in the individualized selection of treatments for each patient.

The present study has several limitations. First of all, although we conducted simulation studies to evaluate the proposed model in terms of parameter recovery and convergence, an application of the model to empirical data is much needed. An immediate next step of the current study is to apply the multilevel HMM to data collected from online learning programs, to assess the fit of the proposed model to real data, and to verify that the model parameters have meaningful practical interpretations.

Secondly, we only considered one target skill per instructional material. We could, however, imagine materials that can simultaneously teach students multiple skills. If we assume conditional independence between the transitions on each skill, then the current model can be straightforwardly extended to the situation of multiple target skills, where the probability of a pattern-wise transition is simply the product of the probabilities of the attribute-wise transitions. However, if the assumption of conditional independence of attribute-wise transitions is relaxed, the learning model will be much more complex. In that case, the probability of a pattern-wise transition will no longer be the product of the attribute-wise transition probabilities, and a learning model will need to look at covariates contributing to the change from one pattern to another for each pair of attribute patterns. Future research can look into models for patternwise transitions, with learning materials' characteristics as covariates.

Another limitation of the current study is the small number of total skills we assumed in the simulation studies. A typical course usually involves a large number of skills, ranging from ten to more than a hundred. However, fitting the current model, or even any simple cognitive diagnosis model under the Bayesian framework with more than ten skills will be computationally intensive. As $K$ increases, the amount of computations required for the MCMC grows quadratically for the sampling of the fixed effect and exponentially for the sampling of the attribute patterns of the subjects at each time point across all $2^{K}$ possible 
candidates. In the case that $K$ is large, we suggest sampling each $\alpha_{i, t, k} \in\{0,1\}$ separately based on $\alpha_{i, t, k^{\prime}} \mathrm{s}$, instead of sampling the entire attribute pattern as a whole. In this way, we could avoid calculating the conditional probabilities of each possible attribute pattern at every iteration for every subject, and instead, for each $i, t$, and $k$, we only need to compute the conditional probabilities of $\alpha_{i, t, k}=0$ and $\alpha_{i, t, k}=1$ given all other parameters. To alleviate the computational burden from sampling of the fixed and random effects, one potential direction is to convert the estimation algorithm of the fixed and random effects from a Metropolis-Hastings sampler to a Gibbs sampler.

Acknowledgements The authors would like to thank Dr. Carolyn Anderson and three anonymous reviewers for their valuable suggestions on the revision of the manuscript.

\section{References}

Chen, Y., Culpepper, S. A., Wang, S., \& Douglas, J. (2017). A hidden Markov model for learning trajectories in cognitive diagnosis with application to spatial rotation skills. Applied Psychological Measurement, 0146621617721250.

Chen, Y., Li, X., Liu, J., \& Ying, Z. (2017). Recommendation system for adaptive learning. Applied Psychological Measurement, 0146621617697959.

Chiu, C.-Y., \& Köhn, H.-F. (2016). The reduced RUM as a logit model: Parameterization and constraints. Psychometrika, 81(2), 350-370.

Collins, L. M., \& Wugalter, S. E. (1992). Latent class models for stage-sequential dynamic latent variables. Multivariate Behavioral Research, 27(1), 131-157.

Culpepper, S. A. (2015). Bayesian estimation of the DINA model with Gibbs sampling. Journal of Educational and Behavioral Statistics, 40(5), 454-476.

dela Torre, J. (2011). The generalized DINA model framework. Psychometrika, 76(2), 179-199.

dela Torre, J., van der Ark, L. A., \& Rossi, G. (2017). Analysis of clinical data from a cognitive diagnosis modeling framework. Measurement and Evaluation in Counseling and Development, $1-16$.

Eddelbuettel, D., François, R., Allaire, J., Chambers, J., Bates, D., \& Ushey, K. (2011). Rcpp: Seamless R and C++ integration. Journal of Statistical Software, 40(8), 1-18.

Gelman, A., Carlin, J. B., Stern, H. S., Dunson, D. B., Vehtari, A., \& Rubin, D. B. (2014). Bayesian data analysis Vol. 2. Boca Raton: CRC Press.

Gelman, A., \& Rubin, D. B. (1992). Inference from iterative simulation using multiple sequences. Statistical Science, 7(4), 457-472.
Gu, Y., \& Xu, G. (2018). The sufficient and necessary condition for the identifiability and estimability of the DINA model. Psychometrika, 1-16.

Gütl, C., Rizzardini, R. H., Chang, V., \& Morales, M. (2014). Attrition in MOOC: Lessons learned from drop-out students. In International Workshop on Learning Technology for Education in Cloud, (pp. 37-48).

Hartz, S. (2002). A Bayesian framework for the unified model for assessing cognitive abilities: Blending theory with practicality (Unpublished doctoral dissertation). Urbana-Champaign: University of Illinois.

Henson, R. A., Templin, J. L., \& Willse, J. T. (2009). Defining a family of cognitive diagnosis models using log-linear models with latent variables. Psychometrika, 74(2), 191-210.

Junker, B. W., \& Sijtsma, K. (2001). Cognitive assessment models with few assumptions, and connections with nonparametric item response theory. Applied Psychological Measurement, 25(3), 258272.

Langeheine, R. (1988). Manifest and latent Markov chain models for categorical panel data. Journal of Educational Statistics, 13(4), 299-312.

Leighton, J. P., Gierl, M. J., \& Hunka, S. M. (2004). The attribute hierarchy method for cognitive assessment: A variation on Tatsuoka's rule-space approach. Journal of Educational Measurement, 41(3), 205-237.

Li, F., Cohen, A., Bottge, B., \& Templin, J. L. (2015). A latent transition analysis model for assessing change in cognitive skills. Educational and Psychological Measurement, 0013164415588946.

Macready, G. B., \& Dayton, C. M. (1977). The use of probabilistic models in the assessment of mastery. Journal of Educational Statistics, 2(2), 99-120.

Oxman, S., \& Wong, W. (2014). White paper: Adaptive learning systems. Integrated Education Solutions.

Rupp, A., Templin, J., \& Henson, R. A. (2010). Diagnostic measurement: Theory, methods, and applications. New York: Guilford Press.

Templin, J. L., \& Henson, R. A. (2006). Measurement of psychological disorders using cognitive diagnosis models. Psychological Methods, 11(3), 287.

Vanlehn, K. (2006). The behavior of tutoring systems. International Journal of Artificial Intelligence in Education, 16(3), 227-265.

von Davier, M. (2008). A general diagnostic model applied to language testing data. British Journal of Mathematical and Statistical Psychology, 61(2), 287-307.

Wang, S., Yang, Y., Culpepper, S. A., \& Douglas, J. A. (2016). Tracking skill acquisition with cognitive diagnosis models: A higher-order, hidden Markov model with covariates. Journal of Educational and Behavioral Statistics, 1076998617719727.

Publisher's note Springer Nature remains neutral with regard to jurisdictional claims in published maps and institutional affiliations. 